\title{
Pre-trained convolutional neural networks as feature extractors toward improved Malaria parasite detection in thin blood smear images
}

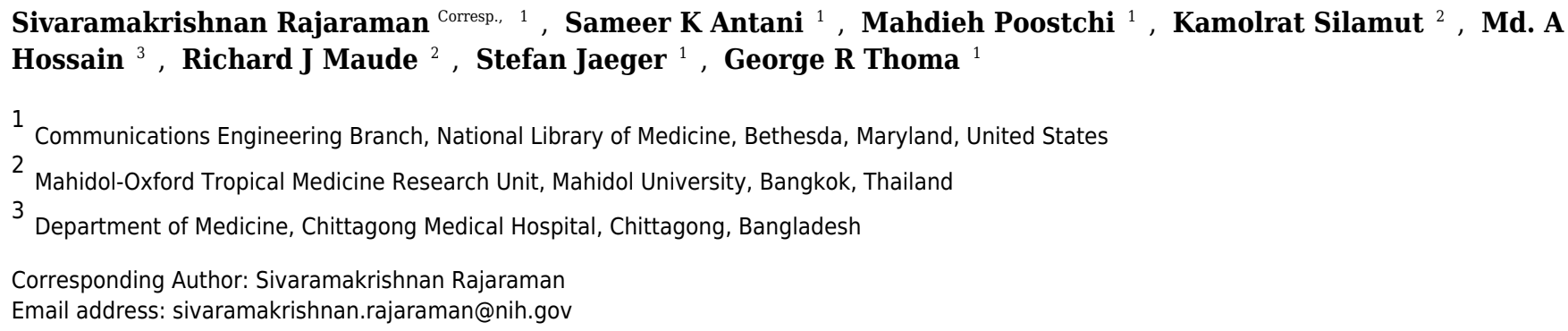

Malaria is a blood disease caused by the Plasmodium parasites transmitted through the bite of female Anopheles mosquito. Microscopists commonly examine thick and thin blood smears to diagnose disease and compute parasitemia. However, their accuracy depends on smear quality and expertise in classifying and counting parasitized and uninfected cells. Such an examination could be arduous for large-scale diagnoses resulting in poor quality. State-of-the-art image-analysis based computer-aided diagnosis (CADx) methods using machine learning $(\mathrm{ML})$ techniques, applied to microscopic images of the smears using hand-engineered features demand expertise in analyzing morphological, textural, and positional variations of the region of interest (ROI). In contrast, Convolutional Neural Networks (CNN), a class of deep learning (DL) models promise highly scalable and superior results with end-to-end feature extraction and classification. Automated malaria screening using DL techniques could, therefore, serve as an effective diagnostic aid. In this study, we evaluate the performance of pre-trained CNN based DL models as feature extractors toward classifying parasitized and uninfected cells to aid in improved disease screening. We experimentally determine the optimal model layers for feature extraction from the underlying data. Statistical validation of the results demonstrates the use of pre-trained CNNs as a promising tool for feature extraction for this purpose. 
1 Pre-trained convolutional neural networks as feature

2 extractors toward improved Malaria parasite detection in thin

3 blood smear images

4 Sivaramakrishnan Rajaraman ${ }^{1}$, Sameer K Antani ${ }^{1}$, Mahdieh Poostchi ${ }^{1}$, Kamolrat Silamut ${ }^{2}$, Md. A Hossain $^{3}$,

5 Richard J Maude ${ }^{2}$, Stefan Jaeger ${ }^{1}$, George R Thoma ${ }^{1}$

$6{ }^{1}$ Communications Engineering Branch, National Library of Medicine, Bethesda, MD 20894, USA

$7 \quad 2$ Mahidol-Oxford Tropical Medicine Research Unit, Mahidol University, Bangkok 10400, Thailand

$8 \quad{ }^{3}$ Department of Medicine, Chittagong Medical Hospital, Chittagong 4203, Bangladesh

9

Corresponding Author:

11 Sivaramakrishnan Rajaraman ${ }^{1}$

12

Email address: sivaramakrishnan.rajaraman@nih.gov

14

15

16

17 
32 Pre-trained convolutional neural networks as feature extractors toward

33

34

35

36

37

\title{
improved Malaria parasite detection in thin blood smear images
} Sivaramakrishnan Rajaraman ${ }^{1}$, Sameer K Antani ${ }^{1}$, Mahdieh Poostchi ${ }^{1}$, Kamolrat Silamut ${ }^{2}$, Md. A Hossain $^{3}$, Richard J Maude ${ }^{2}$, Stefan Jaeger ${ }^{1}$, George R Thoma ${ }^{1}$

${ }^{1}$ Communications Engineering Branch, National Library of Medicine, Bethesda, MD 20894, USA

2 Mahidol-Oxford Tropical Medicine Research Unit, Mahidol University, Bangkok 10400, Thailand

${ }^{3}$ Department of Medicine, Chittagong Medical Hospital, Chittagong 4203, Bangladesh

\begin{abstract}
Malaria is a blood disease caused by the Plasmodium parasites transmitted through the bite of female Anopheles mosquito. Microscopists commonly examine thick and thin blood smears to diagnose disease and compute parasitemia. However, their accuracy depends on smear quality and expertise in classifying and counting parasitized and uninfected cells. Such an examination could be arduous for large-scale diagnoses resulting in poor quality. State-of-the-art image-analysis based computer-aided diagnosis (CADx) methods using machine learning (ML) techniques, applied to microscopic images of the smears using hand-engineered features demand expertise in analyzing morphological, textural, and positional variations of the region of interest (ROI). In contrast, Convolutional Neural Networks (CNN), a class of deep learning (DL) models promise highly scalable and superior results with end-to-end feature extraction and classification. Automated malaria screening using DL techniques could, therefore, serve as an effective diagnostic aid. In this study, we evaluate the performance of pre-trained CNN based DL models as feature extractors toward classifying parasitized and uninfected cells to aid in improved disease screening. We experimentally determine the optimal model layers for feature extraction from the underlying data. Statistical validation of the results demonstrates the use of pre-trained CNNs as a promising tool for feature extraction for this purpose.
\end{abstract}

\section{Introduction}

Malaria is a mosquito-borne blood disease caused by the Plasmodium parasites transmitted through the bite of the female Anopheles mosquito. Different kinds of parasites including P. ovale, $P$. malariae, $P$. vivax and $P$. falciparum infect the humans, however, the effects of $P$. falciparum can be lethal. In 2016, World Health Organization (WHO) reported 212 million instances of the disease across the world (WHO, 2016). Microscopic thick and thin blood smear examinations are the most reliable and commonly used method for disease diagnosis. Thick blood smears assist in detecting the presence of parasites while thin blood smears assist in identifying the species of the parasite causing the infection (Centers for Disease Control and Prevention, 2012). The diagnostic accuracy heavily depends on the human expertise and can be adversely impacted by the interobserver variability and the liability imposed by large-scale diagnoses in diseaseendemic/resource-constrained regions (Mitiku, Mengistu \& Gelaw, 2003). Alternative techniques such as polymerase chain reaction (PCR) and rapid diagnostic tests (RDT) are used, however, PCR 
analysis is limited in its performance (Hommelsheim et al., 2015) and RDTs are less cost-effective in disease-endemic regions (Hawkes, Katsuva \& Masumbuko, 2009). In the process of applying machine learning (ML) methods to medical data analysis, meaningful feature representation lies at the core of their success to accomplish desired results. A majority of image analysis-based computer-aided diagnosis (CADx) software use ML techniques with handengineered features for decision-making (Ross et al., 2006; Das et al., 2013; Poostchi et al., 2018). However, the process demands expertise in analyzing the variability in size, background, angle, and position of the region of interest (ROI) on the images. To overcome challenges of devising hand-engineered features that capture variations in the underlying data, Deep Learning (DL), also known as deep hierarchical learning, is used with significant success (LeCun, Yoshua \& Geoffrey, 2015). DL models use a cascade of layers of non-linear processing units to self-discover hierarchical feature representations in the raw data. Higher-level features are abstracted from lower-level features to aid in learning complex, non-linear decision-making functions, resulting in end-to-end feature extraction and classification (Schmidhuber, 2015). Unlike kernel-based algorithms like Support Vector Machines (SVMs), DL models exhibit improved performance with an increase in data size and computational resources, making them highly scalable (Srivastava et al., 2014).

For images, an important source of information lies in the spatial local correlation among the neighboring pixels/voxels. Convolutional Neural Networks (CNN), a class of DL models are designed to exploit this information through the mechanisms of local receptive fields, shared weights and pooling (Krizhevsky, Sutskever \& Hinton, 2012). In 2012, Alex Krizhevsky proposed AlexNet, a CNN based DL model that won the ImageNet Large Scale Visual Recognition Challenge (ILSVRC) and substantially boosted the performance of CNNs toward classifying natural images (Krizhevsky, Sutskever \& Hinton, 2012). Several representative CNNs like VGGNet (Simonyan \& Zisserman, 2015), GoogLeNet (Szegedy et al., 2014), and ResNet (He et al., 2016) demonstrated significant improvements in succeeding ILSVRC annual challenges. A model named Xception was proposed that uses depth-wise separable convolutions (Chollet, 2016) to outperform the Inception-V3 model (Szegedy et al., 2016) on the ImageNet (Deng et al., 2009) data classification task. A CNN variant called Densely Connected Convolutional Networks (DenseNet) was proposed (Huang et al., 2016) that utilizes a network architecture in which each layer is directly connected to every later layer. The model has achieved noteworthy improvements over the state-of-the-art while using significantly fewer parameters and computations.

The promising performance of CNNs is accompanied by the availability of a huge amount of annotated data. With scarcity for annotated medical imagery, Transfer Learning (TL) methods are used where pre-trained DL models are either fine-tuned on the underlying data or used as feature extractors to aid in visual recognition tasks (Razavian et al., 2014). These models transfer their knowledge gained while learning the generic features from large-scale datasets like ImageNet to the underlying task. The transfer of previously-learned skills to a new situation is generalized, rather than unique to the situation. Since the results published in (Razavian et al., 2014), it is recognized that CNNs trained on large-scale datasets could serve as feature extractors for a wide 
112 range of computer vision tasks to aid in improved performance, as compared to state-of-the-art 113 methods (Bousetouane \& Morris, 2015).

114 At the present, researchers across the world have begun to apply DL tools and obtain promising 115 results in a wide variety of medical image analyses/understanding tasks (Rajaraman et al., 2017; 116 Suzuki, 2017). Literature also reveals studies pertaining to applying DL methods to the task of 117 malaria parasite detection. Dong et al. compared the performance of SVM and pre-trained DL 118 models including LeNet (LeCun et al., 1998), AlexNet, and GoogLeNet toward classifying 119 parasitized and uninfected cells (Dong et al., 2017). Red Blood cells (RBCs) were segmented from 120 thin blood smear images and randomly split into train and test sets. $25 \%$ of the training images

121

122

123

124

125

126

127

128

129

130

131

132

133

134

135

136

137

138

139

140

141

142

143

144

145

146

147

148

149

150

151 were randomly selected to validate the models. Liang et al. proposed a 16-layer CNN toward classifying the uninfected and parasitized cells. Features were extracted using the pre-trained AlexNet and an SVM classifier was trained on the extracted features (Liang et al., 2017). The performance of the proposed model was compared to that of the pre-trained CNN. The study reported that the custom model was more accurate, sensitive and specific than the pre-trained model. Images were resampled to $44 \times 44$ pixel resolution to compensate for the lack of computational resources that would have led to the loss of image resolution. Bibin et al. proposed a 6-layer deep belief network toward malaria parasite detection in peripheral blood smear images (Bibin, Nair \& Punitha, 2017). The authors reported 96.4\% accuracy in the task of classifying a dataset of 4100 cells with randomized train/test splits. Gopakumar et al. employed a customized CNN model for analyzing videos containing a focus stack of the field of views of Leishman stained slide images toward the process of automated parasite detection (Gopakumar et al., 2017). The authors used a customized portable slide scanner and off-the shelf components for data acquisition and demonstrated sensitivity and specificity of $97.06 \%$ and $98.50 \%$ respectively. In summary, existing DL studies have been evaluated on relatively small image sets and/or randomized train/test splits. None of the studies have reported the performance of the predictive models at the patient level. Although the reported outcomes are promising, existing approaches need to substantiate their robustness on a larger set of images with cross-validation studies at the patient level. Evaluation on patient-level provides a more realistic performance evaluation of the predictive models as the images in the independent test set represent truly unseen images for the training process, with no information about staining variations or other artifacts leaking into the training data. This would help to reduce bias and generalization errors. Tests for statistically significant differences in performance would further assist in the process of optimal model selection prior to deployment. It is reasonable to mention that the state-of-the-art still leaves much room for progress in this regard.

In this work, we evaluate the performance of pre-trained CNN based DL models as feature extractors toward classifying the parasitized and uninfected cells to aid in improved disease screening. The important contributions of this work are as follows: (a) presentation of a comparative analysis of the performance of customized and pre-trained DL models as feature extractors toward classifying parasitized and uninfected cells, (b) cross-validating the performance of the predictive models at the patient level to reduce bias and generalization errors, (c) analysis 
152

153

154

155

156

157

158

159

160

161

162

163

164

165

166

167

168

169

170

171

172

173

174

175

176

177

178

179

180

181

182

183

184

185

186

187

188

189

190

and selection of the optimal layer in the pre-trained models to extract features from the underlying data, and (d) testing for the presence/absence of a statistically significant difference in the performance of customized and pre-trained CNN models under study. The following paper is organized as follows: Section 2 elaborates on the materials and methods, Section 3 presents the results, and Section 4 discusses the results and concludes the paper.

\section{Materials and Methods}

\section{Data collection}

To reduce the burden for microscopists in resource-constrained regions and improve diagnostic accuracy, researchers at the Lister Hill National Center for Biomedical Communications (LHNCBC), part of National Library of Medicine (NLM) have developed a mobile application that runs on a standard Android ${ }^{\circledR}$ smartphone attached to a conventional light microscope (Poostchi et al., 2018). Giemsa-stained thin blood smear slides from 150 P. falciparum-infected and 50 healthy patients were collected and photographed at Chittagong Medical College Hospital, Bangladesh. The smartphone's built-in camera acquired images of slides for each microscopic field of view. The images were manually annotated by an expert slide reader at the Mahidol-Oxford Tropical Medicine Research Unit in Bangkok, Thailand. The de-identified images and annotations are archived at NLM (IRB\#12972). We applied a level-set based algorithm to detect and segment the red blood cells (Poostchi et al., 2018).

\section{Cross-validation studies}

The dataset consists of 27,558 cell images with equal instances of parasitized and uninfected cells. Positive samples contained the Plasmodium and negative samples contained no Plasmodium but other types of objects including staining artifacts/impurities. We evaluated the predictive models through five-fold cross-validation. Cross-validation has been performed at the patient level to ensure alleviating model biasing and generalization errors. The count of cells for the different folds is shown in Table 1.

\section{Table 1:}

\section{Data for cross-validation studies.}

The images were re-sampled to $100 \times 100,224 \times 224,227 \times 227$ and $299 \times 299$ pixel resolutions to suit the input requirements of customized and pre-trained CNNs and normalized to assist in faster convergence. The models were trained and tested on a Windows ${ }^{\circledR}$ system with Intel ${ }^{\circledR}$ Xeon ${ }^{\circledR}$ CPU E5-2640v3 2.60-GHz processor, 1 TB HDD, 16 GB RAM, a CUDA-enabled Nvidia ${ }^{\circledR}$ GTX 1080 Ti 11GB graphical processing unit (GPU), Matlab ${ }^{\circledR}$ R2017b, Python ${ }^{\circledR} 3.6 .3$, Keras ${ }^{\circledR} 2.1 .1$ with Tensorflow $^{\circledR}$ 1.4.0 backend, and CUDA 8.0/cuDNN 5.1 dependencies for GPU acceleration.

\section{Customized model configuration}


191 We also evaluated the performance of a customized, sequential CNN in the task of classifying

192

193

194

195

196

197

198

199

200

201

202

203

204

205

206

207

208

209

210

211

212

213

214

215

216

217

218

219

220

221

222

223

224

225

226

227

228

229

230

parasitized and uninfected cells toward disease screening. We propose a sequential CNN as shown in Fig. 1, similar to the architecture that LeCun et al. advocated for image classification (LeCun \& Bengio, 1995).

\section{Figure 1: Architecture of the customized model.}

The proposed CNN has three convolutional layers and two fully connected layers. The input to the model constitutes segmented cells of $100 \times 100 \times 3$ pixel resolution. The convolutional layers use $3 \times 3$ filters with 2 pixel strides. The first and second convolutional layers have 32 filters and the third convolutional layer has 64 filters. The sandwich design of convolutional/rectified linear units (ReLU) and proper weight initialization enhances the learning process (Shang et al., 2016). Maxpooling layers with a pooling window of $2 \times 2$ and 2 pixel strides follow the convolutional layers for summarizing the outputs of neighboring neuronal groups in the feature maps. The pooled output of the third convolutional layer is fed to the first fully-connected layer that has 64 neurons, and the second fully connected layer feeds into the Softmax classifier. Dropout regularization (Srivastava et al., 2014) with a dropout ratio of 0.5 is applied to outputs of the first fully connected layer. The model is trained by optimizing the multinomial logistic regression objective using stochastic gradient descent (SGD) (LeCun, Bengio \& Hinton, 2015) and Nesterov's momentum (Botev, Lever \& Barber, 2017). The customized model is optimized for hyper-parameters by a randomized grid search method (Bergstra \& Bengio, 2012). We initialized search ranges to be [1e7 5e-2], [0.8 0.99] and [1e-10 1e-2] for the learning rate, stochastic gradient descent (SGD) and L2-regularization parameters, respectively. We evaluated the performance of the customized model in terms of accuracy, AUC, sensitivity, specificity, F1-score (Lipton, Elkan \& Naryanaswamy, 2014) and Matthews correlation coefficient (MCC) (Matthews, 1975).

\section{Feature extraction using pre-trained models}

We evaluated the performance of pre-trained CNNs including AlexNet (winner of ILSVRC 2012), VGG-16 (winner of ILSVRC's localization task in 2014), Xception, ResNet-50 (winner of ILSVRC 2015) and DenseNet-121 (winner of the best paper award in CVPR 2017) toward extracting the features from the parasitized and uninfected cells. The models were optimized for hyper-parameters by the randomized grid search method. We initialized search ranges to be [1e-5 5e-2], [0.8 0.99] and [1e-10 1e-2] for the learning rate, Nesterov's accelerated stochastic gradient descent (SGD) and L2-regularization parameters, respectively. We instantiated the convolutional part of the pre-trained CNNs and trained a fully-connected model with dropout (dropout ratio of 0.5 ) on top of the extracted features. We also empirically determined the optimal layer for feature extraction to aid in improved classification. We evaluated the performance of the pre-trained CNNs in terms of accuracy, AUC, sensitivity, specificity, F1-score, and MCC. The model architecture and weights for the pre-trained CNNs were downloaded from GitHub repositories (Chollet; Yu, 2016). 


\section{Statistical analysis}

233

234

235

236

237

238

239

240

241

242

243

244

245

246

247

248

249

250

251

252

253

254

255

256

257

258

259

260

261

262

263

264

265

266

267

268

269

270

We performed statistical analyses to choose the best model for deployment. Statistical methods like one-way analysis of variance (ANOVA) are used to determine the presence or absence of a statistically significant difference between the means of three or more individual, unrelated groups (Rossi, 1987). One-way ANOVA tests the null hypothesis (H0) given by $\mathrm{H} 0: \mu_{1}=\mu_{2}=\ldots=\mu_{\mathrm{k}}$ where $\mu=$ mean of parameters for the individual groups and $\mathrm{k}=$ total number of groups. If a statistically significant result is returned by the test, $\mathrm{H} 0$ is rejected and the alternative hypothesis (H1) is accepted to infer that a statistically significant difference exists between the means of at least two groups under study. However, it would be appropriate to use this parametric test only when the underlying data satisfies the assumptions of independence of observations, absence of significant outliers, normality of data and homogeneity of variances (Daya, 2003). When the conditions are violated, a non-parametric alternative like Kruskal-Wallis H test (also called the one-way ANOVA on ranks) could be used (Vargha, Delaney \& Vargha, 1998). This is an omnibus test that couldn't identify the specific groups that demonstrate statistically significant differences in their mean values. A post-hoc analysis is needed to identify these groups that demonstrate statistically significant differences (Kucuk et al., 2016). We performed Shapiro-Wilk test (Royston, 1992) to check for data normality and Levene's statistic test (Gastwirth, Gel \& Miao, 2009) to study the homogeneity of variances for the performance metrics for the different models under study. Statistical analyses were performed using IBM $^{\circledR}$ SPSS $^{\circledR}$ statistical package (IBM Corp. Released, 2015).

\section{Results}

\section{Cell segmentation and detection}

We applied a level-set based algorithm to detect and segment the red blood cells as shown in Fig. 2. The first step is the cell detection where we applied a multi-scale Laplacian of Gaussian (LoG) filter to detect centroids of individual RBCs.

\section{Figure 2: RBC detection and segmentation using iterative voting and level sets.}

(A) Input image. (B) Initial cell detection using iterative voting. (C) Final RBC segmentation mask. (D) Segmentation results superimposed on the original image.

The generated markers are used to segment the cells within a level set active contour framework to confine the evolving contour to the cell boundary. Morphology opening operation is applied as post-processing to remove false detected objects such as staining artifacts using average cell size. White blood cells (WBCs) are filtered out using a one-to-one correspondence based on cell ground-truth annotations since WBCs are not ROIs for this work. We have evaluated our cell detection based on the manual point-wise annotation of infected and uninfected cells. To do so, we applied a one-to-one point matching scheme: For each segmented cell, we checked the number of manual ground-truth points in the segmented cell region. If there is exactly one point in the 
271 region, we counted this as a true positive (TP). If there is no point in the region, we counted this 272 as a false positive (FP). If there is more than one point in the region, we considered this as an 273 under-segmentation or false negative (FN). These counts then allowed us to compute the presented 274 values for positive predictive value (PPV), sensitivity and F1-score. For cell detection, we obtained a PPV of 0.944 , sensitivity of 0.962 and F1-score of 0.952.

276

\section{Performance metrics evaluation}

For the customized and pre-trained models, we empirically determined the optimum value to be 0.9 and 1e-6 for the SGD momentum and L2-regularization, respectively. For the learning rate, we determined the optimum value to be $1 \mathrm{e}-5$ and $1 \mathrm{e}-6$ for the customized and pre-trained CNNs respectively. The second fully connected layer from AlexNet, VGG-16 and the last layer before the final classification layer from Xception, ResNet-50, and DenseNet-121 were selected for feature extraction. Table 2 lists the performance metrics achieved by the models in the process of classifying parasitized and uninfected cells. We also evaluated the performance of pre-trained CNNs by extracting features from different layers in the process of identifying the optimal layer for feature extraction from the underlying data. The naming conventions for these layers are based on the models obtained from Keras ${ }^{\circledR}$ DL library.

Table 2:

290

291

Layers that gave the best values for the performance metrics are listed in Table 3. Table 4 shows the results obtained by extracting the features from the optimal layers toward classifying the parasitized and uninfected cells.

Table 3:

298

While performing statistical analyses, we observed that the results of Shapiro-Wilk test were statistically significant for all the performance metrics $(p<0.05)$ to signify that the normality of data has been violated. For this reason, we opted to use the non-parametric Kruskal-Wallis H test. The consolidated results of Kruskal-Wallis $\mathrm{H}$ and post-hoc analyses are given in Table 5. We observed that, in terms of accuracy, there existed a statistically significant difference in performance between the different CNNs $\left(\chi^{2}(5)=15.508, p=0.008\right)$. Post-hoc tests further revealed that the statistically significant difference existed between the pre-trained Xception, was observed $\left(\chi^{2}(5)=18.958, p=0.002\right)$ in the performance between Xception, ResNet-50, VGG- 
311 16, and DenseNet-121. Similar results were observed for the F1-score $\left(\chi^{2}(5)=14.798, p=0.011\right)$ 312 and $\operatorname{MCC}\left(\chi^{2}(5)=14.487, p=0.013\right)$. No statistically significant difference was observed across 313 the models in terms of sensitivity $\left(\chi^{2}(5)=5.518, p=0.356\right)$ and specificity $\left(\chi^{2}(5)=6.639, p=\right.$ 314 0.249). However, ResNet-50 obtained the highest mean ranks for accuracy, specificity, F1-score, 315 and MCC.

316

317

\section{Table 5:}

\section{Consolidated results of Kruskal-Wallis $H$ and post-hoc tests.}

\section{Discussions and conclusion}

The customized model converged to an optimal solution due to hyper-parameter optimization, implicit regularization imposed by smaller convolutional filter sizes and aggressive dropouts in the fully connected layers. Usage of L2 regularization reduced the effect of model overfitting and converging to a better solution (Simonyan \& Zisserman, 2015). features like blobs, edges, and colors that are abstracted by the deeper layers to form higher level features to present a more affluent image representation (Zeiler \& Fergus, 2014). Studies from the literature reveal that while using pre-trained CNNs for feature extraction, the features are extracted from the layer, right before the classification layer (Razavian et al., 2014). For this reason, we extracted the features from the second fully connected layer for AlexNet and VGG-16 and the last layer before the final classification layer from Xception, ResNet-50, and DenseNet-121 models.

333

334

335

336

337

338

339

340

341

342

343

344

345

346

347

348

349

350 We observed from the patient-level cross-validation studies (Table 2) that ResNet-50 outperformed the customized and other pre-trained CNNs in all performance metrics toward the task of classifying parasitized and uninfected cells. Literature studies reveal that DenseNet-121 outperformed ResNets and other pre-trained CNNs in the ImageNet data classification task (Huang et al., 2016). In our case, for the binary task of classifying parasitized and uninfected cells, the variability in data is several orders of magnitude smaller, the top layers of deep CNNs like DenseNet-121 are probably too specialized, progressively more complex and not the best candidate to re-use for the task of our interest. For this reason, we evaluated the performance of pre-trained CNNs by extracting features from different layers in the process of identifying the optimal layer for feature extraction from the underlying data (Table 3). In the process, we observed that for the pre-trained CNNs, the performance of the layer before the classification layer was degraded compared to the other layers. In contrast to the results shown in Table 2, DenseNet-121 achieved the best values for sensitivity but demonstrated similar AUC values as ResNet-50 and VGG-16 (Table 4). Both VGG-16 and ResNet-50 were equally accurate and demonstrated equal values for AUC and F1-score. However, ResNet-50 was highly specific, demonstrated high MCC and performed relatively better than the other models under study. These results demonstrate that the final layer of pre-trained CNNs is not always optimal for extracting the features from the underlying data. In our study, features from shallow layers performed better than deep features to aid in improved classification of parasitized and uninfected cells. Literature studies reveal that 
351 MCC is an informative single score to evaluate the performance of a binary classifier in a confusion 352 matrix context (Chicco, 2017). In this regard, ResNet-50 demonstrated statistically significant 353 MCC metrics as compared to the other models. The consolidated results demonstrated that the pre354 trained ResNet-50 relatively outperformed the other models under study toward classifying the 355 parasitized and uninfected cells.

356 While performing Kruskal-Wallis $\mathrm{H}$ and post-hoc analyses, we observed that the pre-trained 357 ResNet-50 obtained the highest mean ranks for accuracy, specificity, F1-score, and MCC. If we 358 were looking to select a model based on a balance between PPV and sensitivity as demonstrated by the F1-score, we could observe that the pre-trained ResNet-50 outperformed the other models under study. We have demonstrated the performance of the models in terms of mean $(\mu)$ and standard deviation $(\sigma)$ to present a measure of the dispersion in the performance metrics. The pretrained ResNet-50 outperformed the other models by achieving $0.947 \pm 0.015$ sensitivity and

364

365

366

367

368

369

370

371

372

373

374

375

376

377

378

379

380

381

382

383

384

385

386

387

388

389 $0.972 \pm 0.10$ specificity. Statistical analyses show that the predictive model could capture all observations within three standard deviations from the mean $[-3 \sigma 3 \sigma]$, i.e., the model could exhibit sensitivity and specificity in the range [0.902 0.992] and [0.942 1.00] respectively. However, our study is focused on disease screening, therefore, the sensitivity metric carries significance. We also determined the number of RBCs be analyzed by the proposed model to confidentially return a positive test result. We used the epiR tools for the Analysis of Epidemiological Data (Stevenson et al., 2015) for these computations. The number of cells needed to diagnose (NND) is defined as the number of RBCs to be tested to yield a correct positive test. Youden's index gives a measure of the performance of the model, the value ranges from -1 to +1 with values closer to 1 for higher values of sensitivity and specificity. With a confidence level (CI) of $0.95(p<0.05)$, we found that $11 \mathrm{RBCs}$ need to be tested to return 10 positive results.

To our knowledge, we could find no comparable literature that performed cross-validation studies at the patient level, with a large-scale clinical dataset for the underlying task. For this reason, we also performed cross-validation studies at the cell level and compared with the state-of-the-art (Table 6).

\section{Table 6:}

\section{Comparison with the state-of-the-art literature.}

In the process, we found that the pre-trained ResNet-50 outperformed the state-of-the-art in all performance metrics. Das et al. achieved similar values for sensitivity with a small-scale dataset but demonstrated sub-optimal specificity. The lack of performance at the patient level is attributed to the staining variations between patients. We observed that it is harder for the classifier to learn the different stains, which indicates that we may need to acquire more images with different staining colors for training or apply color normalization techniques. However, by validating the predictive models at the patient-level, which we believe simulate real-world conditions, we ensure getting rid of bias, reduce overfitting and generalization errors toward optimal model deployment. 
390

391

392

393

394

395

396

397

398

399

400

401

402

403

404

405

406

407

408

409

410

411

412

413

414

415

416

417

418

419

420

421

422

423

424

425

426

427

428

429

We are currently performing pilot studies in deploying the customized and pre-trained DL models into mobile devices and analyzing the performances. From the literature studies, we observed that we could either opt to both train/predict on the mobile device or to train the model offline and import to the mobile device to predict on the independent test data (Howard et al., 2017). Currently, Android and IOS ML libraries (like CoreMLStudio) offer the flexibility for dynamic allocation of CPU and GPU based on the computational cost, thus, memory allocation probably doesn't seem to an issue while deploying deep CNN models. From our pilot studies, we observed that the proposed model occupied only $96 \mathrm{MB}$ and took less RAM to do prediction on the test data. The deployed model could serve as triage, minimize delays in disease-endemic/resource-constrained settings.

\section{References}

Bergstra J., Bengio Y. 2012. Random Search for Hyper-Parameter Optimization. Journal of Machine Learning Research 13:281-305. DOI: 10.1162/153244303322533223.

Bibin D., Nair MS., Punitha P. 2017. Malaria Parasite Detection from Peripheral Blood Smear Images Using Deep Belief Networks. IEEE Access 5:9099-9108. DOI: 10.1109/ACCESS.2017.2705642.

Botev A., Lever G., Barber D. 2017. Nesterov's accelerated gradient and momentum as approximations to regularised update descent. In: Proceedings of the International Joint Conference on Neural Networks. 1899-1903. DOI: 10.1109/IJCNN.2017.7966082.

Bousetouane F., Morris B. 2015. Off-the-shelf CNN features for fine-grained classification of vessels in a maritime environment. In: Lecture Notes in Computer Science (including subseries Lecture Notes in Artificial Intelligence and Lecture Notes in Bioinformatics). 379-388. DOI: 10.1007/978-3-319-27863-6_35.

Centers for Disease Control and Prevention. 2012. CDC - Malaria. Available at http://www.cdc.gov/malaria/about/biology/. DOI: 10.1371/journal.pmed.0030473.

Chicco D. 2017. Ten quick tips for machine learning in computational biology. BioData Mining 10. DOI: 10.1186/s13040-017-0155-3.

Chollet F. Deep Learning Models. Available at https://github.com/fchollet/deep-learning-models (accessed February 2, 2017).

Chollet F. 2016. Xception: Deep Learning with Separable Convolutions. arXiv preprint arXiv:1610.02357:1-14.

Das DK., Ghosh M., Pal M., Maiti AK., Chakraborty C. 2013. Machine learning approach for automated screening of malaria parasite using light microscopic images. Micron 45:97-106. DOI: 10.1016/j.micron.2012.11.002.

Daya S. 2003. One-way analysis of variance. Evidence-based Obstetrics and Gynecology 5:153155. DOI: 10.1016/j.ebobgyn.2003.11.001.

Deng J., Dong W., Socher R., Li LJ., Li K., Fei-Fei L. 2009. ImageNet: A large-scale hierarchical image database. In: 2009 IEEE Conference on Computer Vision and Pattern Recognition. 248255. DOI: 10.1109/CVPRW.2009.5206848. 
430 Dong Y., Jiang Z., Shen H., David Pan W., Williams LA., Reddy VVB., Benjamin WH., Bryan 431 AW. 2017. Evaluations of deep convolutional neural networks for automatic identification of 432 malaria infected cells. In: 2017 IEEE EMBS International Conference on Biomedical and Health 433 Informatics, BHI 2017. 101-104. DOI: 10.1109/BHI.2017.7897215.

434 Gastwirth JL., Gel YR., Miao W. 2009. The Impact of Levene's Test of Equality of Variances on 435 Statistical Theory and Practice. Statistical Science 24:343-360. DOI: 10.1214/09-STS301.

436 Gopakumar GP., Swetha M., Sai Siva G., Sai Subrahmanyam GRK. 2017. Convolutional neural 437 network-based malaria diagnosis from focus stack of blood smear images acquired using custom438 built slide scanner. Journal of Biophotonics:e201700003. DOI: 10.1002/jbio.201700003.

439 Hawkes M., Katsuva J., Masumbuko CK. 2009. Use and limitations of malaria rapid diagnostic 440 testing by community health workers in war-torn Democratic Republic of Congo. Malaria Journal 441 8:308. DOI: 10.1186/1475-2875-8-308.

442 He K., Zhang X., Ren S., Sun J. 2016. Deep Residual Learning for Image Recognition. In: 2016 443 IEEE Conference on Computer Vision and Pattern Recognition (CVPR). 770-778. DOI: 444 10.1109/CVPR.2016.90.

445 Hommelsheim CM., Frantzeskakis L., Huang M., Ülker B. 2015. PCR amplification of repetitive 446 DNA: a limitation to genome editing technologies and many other applications. Scientific Reports 447 4:5052. DOI: 10.1038/srep05052.

448 Howard AG., Zhu M., Chen B., Kalenichenko D., Wang W., Weyand T., Andreetto M., Adam H. 449 2017. MobileNets: Efficient Convolutional Neural Networks for Mobile Vision Applications. 450 ArXiv:9. DOI: arXiv:1704.04861.

451 Huang G., Liu Z., Weinberger KQ., van der Maaten L. 2016. Densely Connected Convolutional 452 Networks. DOI: 10.1109/CVPR.2017.243.

453 Krizhevsky A., Sutskever I., Hinton GE. 2012. ImageNet Classification with Deep Convolutional 454 Neural Networks. In: Advances In Neural Information Processing Systems. 1-9. DOI: 455 http://dx.doi.org/10.1016/j.protcy.2014.09.007.

456 Kucuk U., Eyuboglu M., Kucuk HO., Degirmencioglu G. 2016. Importance of using proper post 457 hoc test with ANOVA. International Journal of Cardiology 209:346. DOI: 458 10.1016/j.ijcard.2015.11.061.

459 LeCun Y., Bengio Y. 1995. Convolutional networks for images, speech, and time series. The 460 handbook of brain theory and neural networks 3361:255-258. DOI: 461 10.1109/IJCNN.2004.1381049.

462 LeCun Y., Bottou L., Bengio Y., Haffner P. 1998. Gradient-based learning applied to document 463 recognition. Proceedings of the IEEE 86:2278-2323. DOI: 10.1109/5.726791.

464 LeCun Y., Yoshua B., Geoffrey H. 2015. Deep learning. Nature 521:436-444. DOI: 46510.1038 /nature14539.

466 Liang Z., Powell A., Ersoy I., Poostchi M., Silamut K., Palaniappan K., Guo P., Hossain MA., 467 Sameer A., Maude RJ., Huang JX., Jaeger S., Thoma G. 2017. CNN-based image analysis for 468 malaria diagnosis. In: Proceedings - 2016 IEEE International Conference on Bioinformatics and 469 Biomedicine, BIBM 2016. 493-496. DOI: 10.1109/BIBM.2016.7822567. 
470 Lipton ZC., Elkan C., Naryanaswamy B. 2014. Optimal thresholding of classifiers to maximize 471 F1 measure. In: Lecture Notes in Computer Science (including subseries Lecture Notes in 472 Artificial Intelligence and Lecture Notes in Bioinformatics). 225-239. DOI: 10.1007/978-3-662473 44851-9_15.

474 Matthews BW. 1975. Comparison of the predicted and observed secondary structure of T4 phage 475 lysozyme. BBA - Protein Structure 405:442-451. DOI: 10.1016/0005-2795(75)90109-9.

476 Mitiku K., Mengistu G., Gelaw B. 2003. The reliability of blood film examination for malaria at 477 the peripheral health unit. Ethiop.J.Health Dev. 17:197-204.

478 Poostchi M., Silamut K., Maude R., Jaeger S., Thoma G. 2018. Image analysis and machine 479 learning for detecting malaria. Translational Research. DOI: 10.1016/j.trsl.2017.12.004.

480 Razavian AS., Azizpour H., Sullivan J., Carlsson S. 2014. CNN features off-the-shelf: An 481 astounding baseline for recognition. In: IEEE Computer Society Conference on Computer Vision 482 and Pattern Recognition Workshops. 512-519. DOI: 10.1109/CVPRW.2014.131.

484 Rajaraman S., Antani S., Xue Z., Candemir S., Jaeger S. 2017. Visualizing abnormalities in chest radiographs through salient network activations in Deep Learning. In: Life Sciences Conference (LSC), 2017 IEEE. Sydney, NSW, Australia: IEEE, 71-74. DOI: 10.1109/LSC.2017.8268146.

486 Ross NE., Pritchard CJ., Rubin DM., Dusé AG. 2006. Automated image processing method for the diagnosis and classification of malaria on thin blood smears. Medical \& biological engineering \& computing 44:427-436. DOI: 10.1007/s11517-006-0044-2.

489 Rossi JS. 1987. One-Way Anova from Summary Statistics. Educational and Psychological 490 Measurement 47:37-38. DOI: 10.1177/0013164487471004.

491

492

493

494

495

496

497

498

499

500

501

502

503

504

505

506

507

508

Royston P. 1992. Approximating the Shapiro-Wilk W-test for non-normality. Statistics and Computing 2:117-119. DOI: 10.1007/BF01891203.

Schmidhuber J. 2015. Deep Learning in neural networks: An overview. Neural Networks 61:85117. DOI: $10.1016 /$ j.neunet.2014.09.003.

Shang W., Sohn K., Almeida D., Lee H. 2016. Understanding and Improving Convolutional Neural Networks via Concatenated Rectified Linear Units. In: Proceedings of 33rd International Conference on Machine Learning (ICML2016).

Simonyan K., Zisserman A. 2015. Very Deep Convolutional Networks for Large-Scale Image Recognition. International Conference on Learning Representations (ICRL). DOI: 10.1016/j.infsof.2008.09.005.

Srivastava N., Hinton G., Krizhevsky A., Sutskever I., Salakhutdinov R. 2014. Dropout: A Simple Way to Prevent Neural Networks from Overfitting. Journal of Machine Learning Research 15:1929-1958. DOI: 10.1214/12-AOS1000.

Stevenson M., Nunes T., Heuer C., Marshall J., Sanchez J., Thornton R., Reiczigel J., RobisonCox J., Sebastiani P., Solymos P., Yoshida K., Firestone S. 2015. Tools for the Analysis of Epidemiological Data R package version 0.9-62. CRAN.R-project.org.

Suzuki K. 2017. Overview of deep learning in medical imaging. Radiological Physics and Technology 10:257-273. DOI: 10.1007/s12194-017-0406-5. 
509 Szegedy C., Liu W., Jia Y., Sermanet P. 2014. Going deeper with convolutions. arXiv preprint 510 arXiv: 1409.4842:1-9. DOI: 10.1109/CVPR.2015.7298594.

511 Szegedy C., Vanhoucke V., Ioffe S., Shlens J., Wojna Z. 2016. Rethinking the Inception 512 Architecture for Computer Vision. Proceedings of the IEEE Computer Society Conference on 513 Computer Vision and Pattern Recognition (CVPR):2818-2826. DOI: 10.1002/2014GB005021.

514 Vargha A., Delaney HD., Vargha A. 1998. The Kruskal-Wallis Test and Stochastic Homogeneity. 515 Journal of Educational and Behavioral Statistics 23:170. DOI: 10.2307/1165320.

516 WHO. 2016. World Malaria Report. Available at 517 http://apps.who.int/iris/bitstream/10665/252038/1/9789241511711-eng.pdf?ua=1 (accessed 518 January 4, 2017). DOI: 10.4135/9781452276151.n221.

519 Yu F. 2016.Fine-tune CNN in Keras. Available at https://github.com/flyyufelix/cnn_finetune 520 (accessed October 2, 2017).

521 Zeiler MD., Fergus R. 2014. Visualizing and understanding convolutional networks. In: Lecture 522 Notes in Computer Science (including subseries Lecture Notes in Artificial Intelligence and 523 Lecture Notes in Bioinformatics). 818-833. DOI: 10.1007/978-3-319-10590-1_53. 
Figure 1

Architecture of the customized model. 


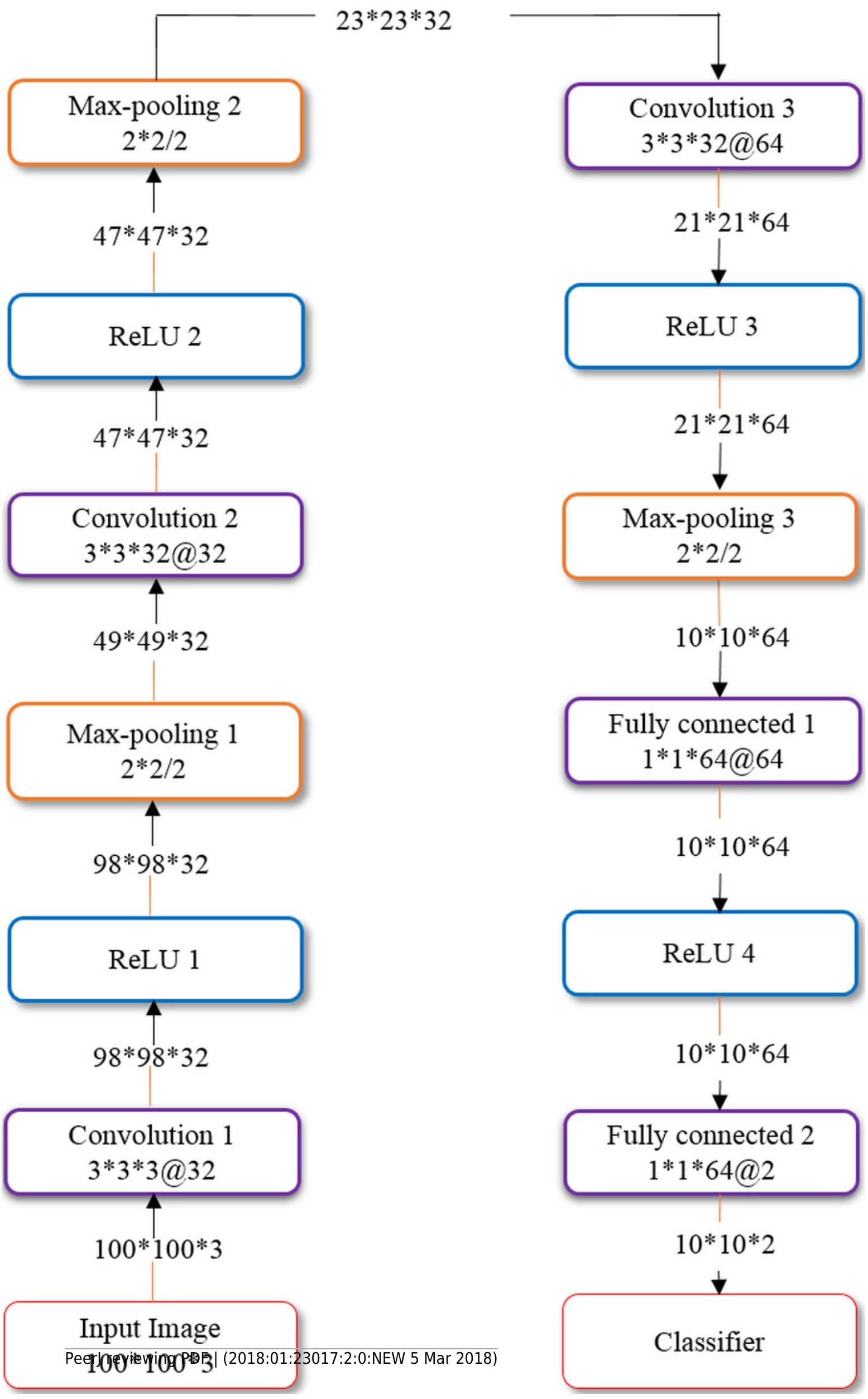


Figure 2

RBC detection and segmentation using iterative voting and level sets.

(A) Input image. (B) Initial cell detection using iterative voting. (C) Final RBC segmentation mask. (D) Segmentation results superimposed on the original image.

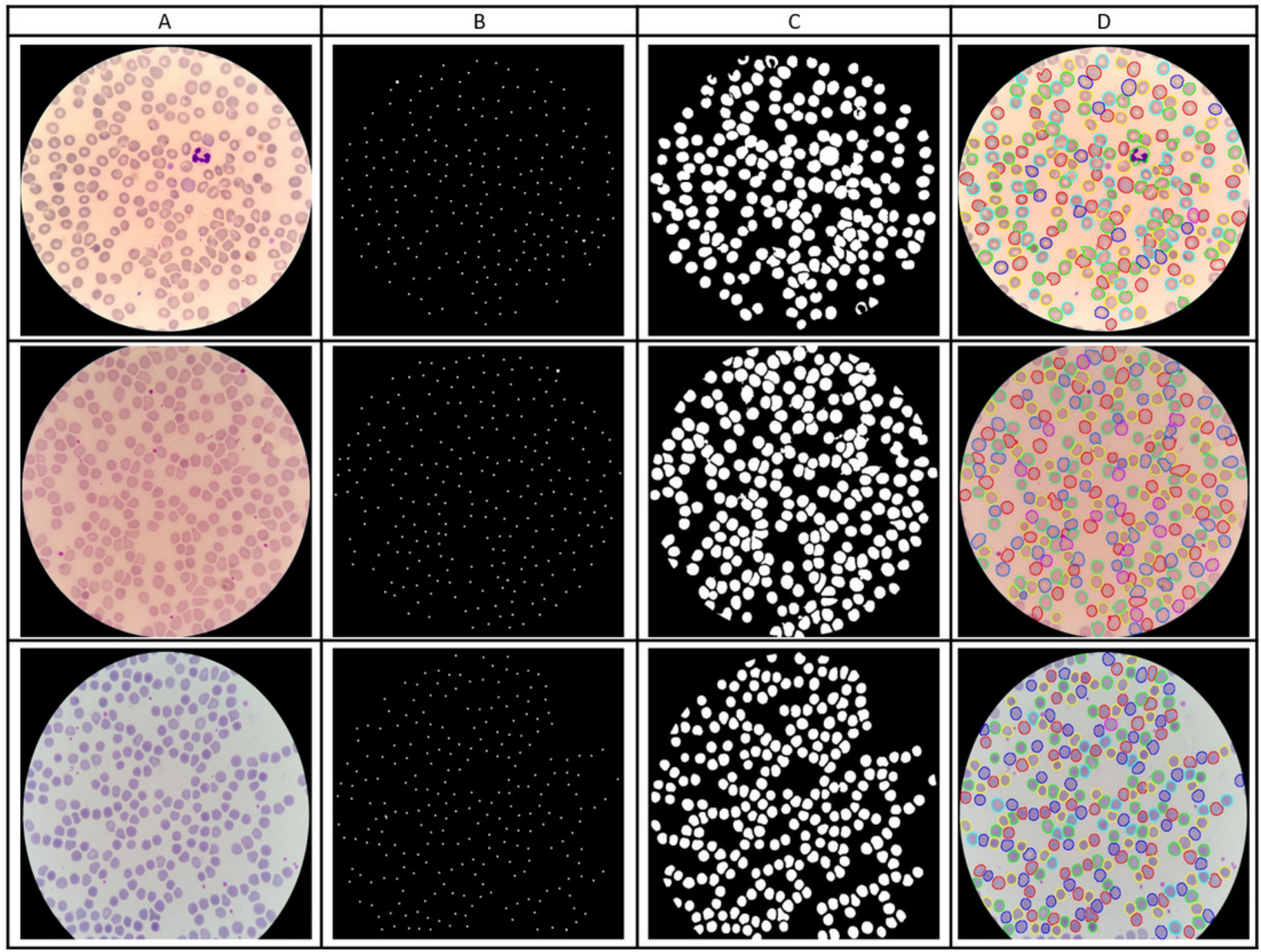


Table $\mathbf{1}$ (on next page)

Data for cross-validation studies. 
1 Table 1:

2 Data for cross-validation studies.

3

\begin{tabular}{|c|c|c|}
\hline Folds & Parasitized & Uninfected \\
\hline 1 & 2756 & 2757 \\
\hline 2 & 2758 & 2758 \\
\hline 3 & 2776 & 2762 \\
\hline 4 & 2832 & 2760 \\
\hline 5 & 2657 & 2742 \\
\hline Total & $\mathbf{1 3 7 7 9}$ & $\mathbf{1 3 7 7 9}$ \\
\hline
\end{tabular}

4

5 
Table 2 (on next page)

Performance metrics. 
1 Table 2:

2 Performance metrics.

3

\begin{tabular}{|c|c|c|c|c|c|c|}
\hline Models & Accuracy & AUC & Sensitivity & Specificity & F1-score & MCC \\
\hline AlexNet & $0.937 \pm 0.012$ & $0.981 \pm 0.007$ & $0.940 \pm 0.017$ & $0.933 \pm 0.034$ & $0.937 \pm 0.011$ & $0.872 \pm 0.024$ \\
\hline VGG-16 & $0.945 \pm 0.015$ & $0.981 \pm 0.007$ & $0.939 \pm 0.022$ & $0.951 \pm 0.019$ & $0.945 \pm 0.016$ & $0.887 \pm 0.030$ \\
\hline ResNet-50 & $\mathbf{0 . 9 5 7} \pm \mathbf{0 . 0 0 7}$ & $\mathbf{0 . 9 9 0} \pm \mathbf{0 . 0 0 4}$ & $\mathbf{0 . 9 4 5} \pm \mathbf{0 . 0 2 0}$ & $\mathbf{0 . 9 6 9} \pm \mathbf{0 . 0 0 9}$ & $\mathbf{0 . 9 5 7} \pm \mathbf{0 . 0 0 8}$ & $\mathbf{0 . 9 1 2} \pm \mathbf{0 . 0 1 4}$ \\
\hline Xception & $0.890 \pm 0.107$ & $0.948 \pm 0.062$ & $0.931 \pm 0.039$ & $0.835 \pm 0.218$ & $0.895 \pm 0.100$ & $0.772 \pm 0.233$ \\
\hline $\begin{array}{c}\text { DenseNet- } \\
121\end{array}$ & $0.931 \pm 0.018$ & $0.976 \pm 0.023$ & $0.942 \pm 0.023$ & $0.926 \pm 0.032$ & $0.931 \pm 0.017$ & $0.894 \pm 0.036$ \\
\hline Customized & $0.940 \pm 0.010$ & $0.979 \pm 0.009$ & $0.931 \pm 0.026$ & $0.951 \pm 0.030$ & $0.941 \pm 0.010$ & $0.880 \pm 0.020$ \\
\hline
\end{tabular}

4

5 


\section{Table $\mathbf{3}$ (on next page)}

Candidate layers giving the best performance. 
1 Table 3:

2 Candidate layers giving the best performance.

3

\begin{tabular}{|c|c|}
\hline Model & Optimal layer \\
\hline AlexNet & fc6 \\
\hline VGG-16 & block5_conv2 \\
\hline ResNet-50 & res5c_branch2c \\
\hline Xception & block14_sepconv1 \\
\hline DenseNet-121 & Conv5_16_x2 \\
\hline
\end{tabular}

4 


\section{Table 4(on next page)}

Performance metrics achieved with feature extraction from optimal layers. 
1 Table 4:

2 Performance metrics achieved with feature extraction from optimal layers.

3

\begin{tabular}{|c|c|c|c|c|c|c|}
\hline Models & Accuracy & AUC & Sensitivity & Specificity & F1-score & MCC \\
\hline AlexNet & $0.944 \pm 0.010$ & $0.983 \pm 0.006$ & $0.947 \pm 0.016$ & $0.941 \pm 0.025$ & $0.944 \pm 0.010$ & $0.886 \pm 0.020$ \\
\hline VGG-16 & $\mathbf{0 . 9 5 9} \pm \mathbf{0 . 0 0 9}$ & $\mathbf{0 . 9 9 1} \pm \mathbf{0 . 0 0 4}$ & $0.949 \pm 0.020$ & $0.969 \pm 0.016$ & $\mathbf{0 . 9 5 9} \pm \mathbf{0 . 0 0 9}$ & $0.916 \pm 0.017$ \\
\hline ResNet-50 & $\mathbf{0 . 9 5 9} \pm \mathbf{0 . 0 0 8}$ & $\mathbf{0 . 9 9 1} \pm \mathbf{0 . 0 0 5}$ & $0.947 \pm 0.015$ & $\mathbf{0 . 9 7 2} \pm \mathbf{0 . 0 1 0}$ & $\mathbf{0 . 9 5 9} \pm \mathbf{0 . 0 0 9}$ & $\mathbf{0 . 9 1 7} \pm \mathbf{0 . 0 1 7}$ \\
\hline Xception & $0.915 \pm 0.005$ & $0.965 \pm 0.019$ & $0.925 \pm 0.039$ & $0.907 \pm 0.120$ & $0.918 \pm 0.042$ & $0.836 \pm 0.088$ \\
\hline $\begin{array}{c}\text { DenseNet- } \\
121\end{array}$ & $0.952 \pm 0.022$ & $\mathbf{0 . 9 9 1} \pm \mathbf{0 . 0 0 4}$ & $\mathbf{0 . 9 6 0} \pm \mathbf{0 . 0 0 9}$ & $0.944 \pm 0.048$ & $0.953 \pm 0.020$ & $0.902 \pm 0.041$ \\
\hline Customized & $0.927 \pm 0.026$ & $0.978 \pm 0.012$ & $0.905 \pm 0.074$ & $0.951 \pm 0.031$ & $0.928 \pm 0.041$ & $0.884 \pm 0.002$ \\
\hline
\end{tabular}

4 


\section{Table 5 (on next page)}

Consolidated results of Kruskal-Wallis $\mathrm{H}$ and post-hoc tests. 
1 Table 5:

2 Consolidated results of Kruskal-Wallis $\mathrm{H}$ and post-hoc tests.

3

\begin{tabular}{|c|c|c|c|c|}
\hline Metric & Kruskal-Wallis H & \multicolumn{2}{|c|}{ Mean ranks } & Post-hoc \\
\hline \multirow[t]{6}{*}{ Accuracy } & \multirow{6}{*}{$\begin{array}{c}\chi^{2}(5)=15.508, p= \\
0.008\end{array}$} & AlexNet & 11.20 & \multirow{6}{*}{$\begin{array}{l}\text { Xception \& ResNet-50 }(p= \\
0.005) \\
\text { Xception \& VGG-16 }(p= \\
0.007) \\
\text { Customized \& ResNet-50 ( } p \\
=0.017)\end{array}$} \\
\hline & & VGG-16 & 22.30 & \\
\hline & & ResNet-50 & 23.00 & \\
\hline & & Xception & 7.20 & \\
\hline & & DenseNet-121 & 19.60 & \\
\hline & & Customized & 9.70 & \\
\hline \multirow[t]{6}{*}{ AUC } & \multirow{6}{*}{$\begin{array}{c}\chi^{2}(5)=18.958, p= \\
0.002\end{array}$} & AlexNet & 13.00 & \multirow{6}{*}{$\begin{array}{c}\text { Xception \& ResNet-50 }(p= \\
0.034) \\
\text { Xception \& VGG-16 }(p= \\
0.030) \\
\text { Xception \& DenseNet-121 }(p \\
=0.014)\end{array}$} \\
\hline & & VGG-16 & 21.70 & \\
\hline & & ResNet-50 & 21.50 & \\
\hline & & Xception & 4.50 & \\
\hline & & DenseNet-121 & 22.90 & \\
\hline & & Customized & 9.40 & \\
\hline \multirow[t]{6}{*}{ Sensitivity } & \multirow{6}{*}{$\begin{array}{c}\chi^{2}(5)=5.518, p= \\
0.356\end{array}$} & AlexNet & 16.20 & \multirow[t]{6}{*}{-} \\
\hline & & VGG-16 & 17.30 & \\
\hline & & ResNet-50 & 15.80 & \\
\hline & & Xception & 11.40 & \\
\hline & & DenseNet-121 & 21.80 & \\
\hline & & Customized & 10.50 & \\
\hline \multirow[t]{6}{*}{ Specificity } & \multirow{6}{*}{$\begin{array}{c}\chi^{2}(5)=6.639, p= \\
0.249\end{array}$} & AlexNet & 9.80 & \multirow[t]{6}{*}{-} \\
\hline & & VGG-16 & 20.70 & \\
\hline & & ResNet-50 & 21.30 & \\
\hline & & Xception & 13.30 & \\
\hline & & DenseNet-121 & 14.10 & \\
\hline & & Customized & 13.80 & \\
\hline \multirow[t]{6}{*}{ F1-score } & \multirow{6}{*}{$\begin{array}{c}\chi^{2}(5)=14.798, p= \\
0.011\end{array}$} & AlexNet & 11.70 & \multirow{6}{*}{$\begin{array}{l}\text { Xception \& ResNet-50 }(p= \\
0.005) \\
\text { Xception \& VGG-16 }(p= \\
0.006) \\
\text { Xception \& DenseNet-121 }(p \\
=0.023)\end{array}$} \\
\hline & & VGG-16 & 22.20 & \\
\hline & & ResNet-50 & 22.60 & \\
\hline & & Xception & 6.90 & \\
\hline & & DenseNet-121 & 19.50 & \\
\hline & & Customized & 10.10 & \\
\hline \multirow[t]{3}{*}{$\mathrm{MCC}$} & \multirow{3}{*}{$\begin{array}{c}\chi^{2}(5)=14.487, p= \\
0.013\end{array}$} & AlexNet & 11.30 & \multirow{3}{*}{$\begin{array}{c}\text { Xception \& ResNet-50 ( } p= \\
0.007) \\
\text { Xception \& VGG-16 ( } p=\end{array}$} \\
\hline & & VGG-16 & 22.30 & \\
\hline & & ResNet-50 & 22.60 & \\
\hline
\end{tabular}




\begin{tabular}{|c|c|c|c|c|}
\hline \multirow{2}{*}{} & Xception & 7.60 & $0.008)$ \\
\cline { 3 - 3 } & DenseNet-121 & 19.40 & $\begin{array}{c}\text { Xception \& DenseNet-121 }(p \\
=0.034)\end{array}$ \\
\cline { 3 - 3 } & Customized & 9.80 & $\begin{array}{c}\text { Customized \& ResNet-50 }(p \\
=0.021\end{array}$ \\
\hline
\end{tabular}

4

5 
Table 6(on next page)

Comparison with the state-of-the-art literature. 
1 Table 6:

2 Comparison with the state-of-the-art literature.

3

\begin{tabular}{|l|l|l|l|l|l|l|}
\hline \multicolumn{1}{|c|}{ Method } & Accuracy & Sensitivity & Specificity & AUC & F1-score & MCC \\
\hline $\begin{array}{l}\text { Proposed } \\
\text { Model (cell } \\
\text { Level) }\end{array}$ & $\mathbf{0 . 9 8 6}$ & $\mathbf{0 . 9 8 1}$ & $\mathbf{0 . 9 9 2}$ & $\mathbf{0 . 9 9 9}$ & $\mathbf{0 . 9 8 7}$ & $\mathbf{0 . 9 7 2}$ \\
\hline $\begin{array}{l}\text { Proposed } \\
\text { Model (patient } \\
\text { level) }\end{array}$ & 0.959 & 0.947 & 0.972 & 0.991 & 0.959 & 0.917 \\
\hline $\begin{array}{l}\text { Gopakumar et } \\
\text { al. } \\
\text { (Gopakumar et } \\
\text { al., 2017) }\end{array}$ & 0.977 & 0.971 & 0.985 & - & & \\
\hline $\begin{array}{l}\text { Bibin et al. } \\
\text { (Bibin, Nair \& } \\
\text { Punitha, 2017) }\end{array}$ & 0.963 & 0.976 & 0.959 & - & - & \\
\hline $\begin{array}{l}\text { Dong et al. } \\
\text { (Dong et al., } \\
\text { 2017) }\end{array}$ & 0.981 & - & & & & \\
\hline $\begin{array}{l}\text { Liang et al. } \\
\text { (Liang et al., } \\
\text { 2017) }\end{array}$ & 0.973 & 0.969 & 0.977 & - & & \\
\hline $\begin{array}{l}\text { Das et al. (Das } \\
\text { et al., 2013) }\end{array}$ & 0.840 & $\mathbf{0 . 9 8 1}$ & 0.689 & - & & \\
\hline $\begin{array}{l}\text { Ross et al. } \\
\text { (Ross et al., } \\
\text { 2006) }\end{array}$ & 0.730 & 0.850 & - & - & & \\
\hline
\end{tabular}

4

5

6

7

8

9 